\title{
Leaflet manual of external beam radiation therapy for hepatocellular carcinoma: a review of the indications, evidences, and clinical trials
}

This article was published in the following Dove Press journal:

OncoTargets and Therapy

\author{
Chai Hong Rim \\ Won Sup Yoon \\ Department of Radiation \\ Oncology, Ansan Hospital, Korea \\ University Medical College, Ansan, \\ Republic of Korea
}

\begin{abstract}
The use of external beam radiation therapy (EBRT) in the treatment of hepatocellular carcinoma (HCC), which was rarely performed due to liver toxicity with a previous technique, has increased. Palliation of portal vein thrombosis, supplementation for insufficient transarterial chemoembolization, and provision of new curative opportunities using stereotactic body radiotherapy are the potential indications for use of EBRT. The mechanism of EBRT treatment, with its radiobiological and physical perspectives, differs from those of conventional medical treatment or surgery. Therefore, understanding the effects of EBRT may be unfamiliar to physicians other than radiation oncologists, especially in the field of HCC, where EBRT has recently begun to be applied. The first objective of this review was to concisely explain the indications for use of EBRT for HCC for all physicians treating HCC. Therefore, this review focuses on the therapeutic outcomes rather than the detailed biological and physical background. We also reviewed recent clinical trials that may extend the indications for use of EBRT. Finally, we reviewed the current clinical practice guidelines for the treatment of HCC and discuss the current recommendations and future perspectives.
\end{abstract}

Keywords: hepatocellular carcinoma, liver neoplasm, external beam radiotherapy, stereotactic body radiotherapy, clinical trials, sorafenib, guidelines

\section{Introduction}

Liver cancer is the second and sixth-leading cause of cancer-related death in men in developing and developed countries, respectively. The incidence and mortality rates of liver cancer demonstrate large regional variations. Liver cancer overwhelmingly occurs in East Asia; the prevalence rate in this region (32 cases/100,000 people/year) is three times higher than those observed in southern Europe and the United States. ${ }^{1}$ Hepatocellular carcinoma (HCC) is the most common type of primary liver cancer, accounting for $70 \%-90 \%$ of these cancers. Chronic hepatitis B infection, which increases the risk of HCC by up to 100-fold, is the most common cause of HCC in East Asia. ${ }^{2,3}$

Southern Europe has the highest incidence of liver cancer in Europe. The Barcelona Clinic of Liver Cancer (BCLC) classification and treatment guidelines, which are currently considered as the standard guidelines for the treatment of $\mathrm{HCC}$, were developed in this region. ${ }^{4}$ These guidelines utilize the specific characteristics of $\mathrm{HCC}$, including liver function, vascular invasion, and performance status, to recommend a single standard treatment for each stage, yielding clinical convenience. Since several randomized studies have shown that sorafenib confers a survival benefit, recent BCLC guidelines have recommended it as a standard treatment option for advanced $\mathrm{HCC} .{ }^{5}$
Correspondence: Won Sup Yoon Department of Radiation Oncology, Ansan Hospital, Korea University, 123 Jeokgeum-ro, Danwon-gu, Ansan, Gyeonggi-do, 15355, Republic of Korea Tel +82 3I 4I 26850

Fax +82314126851

Email irionyws@korea.ac.kr 


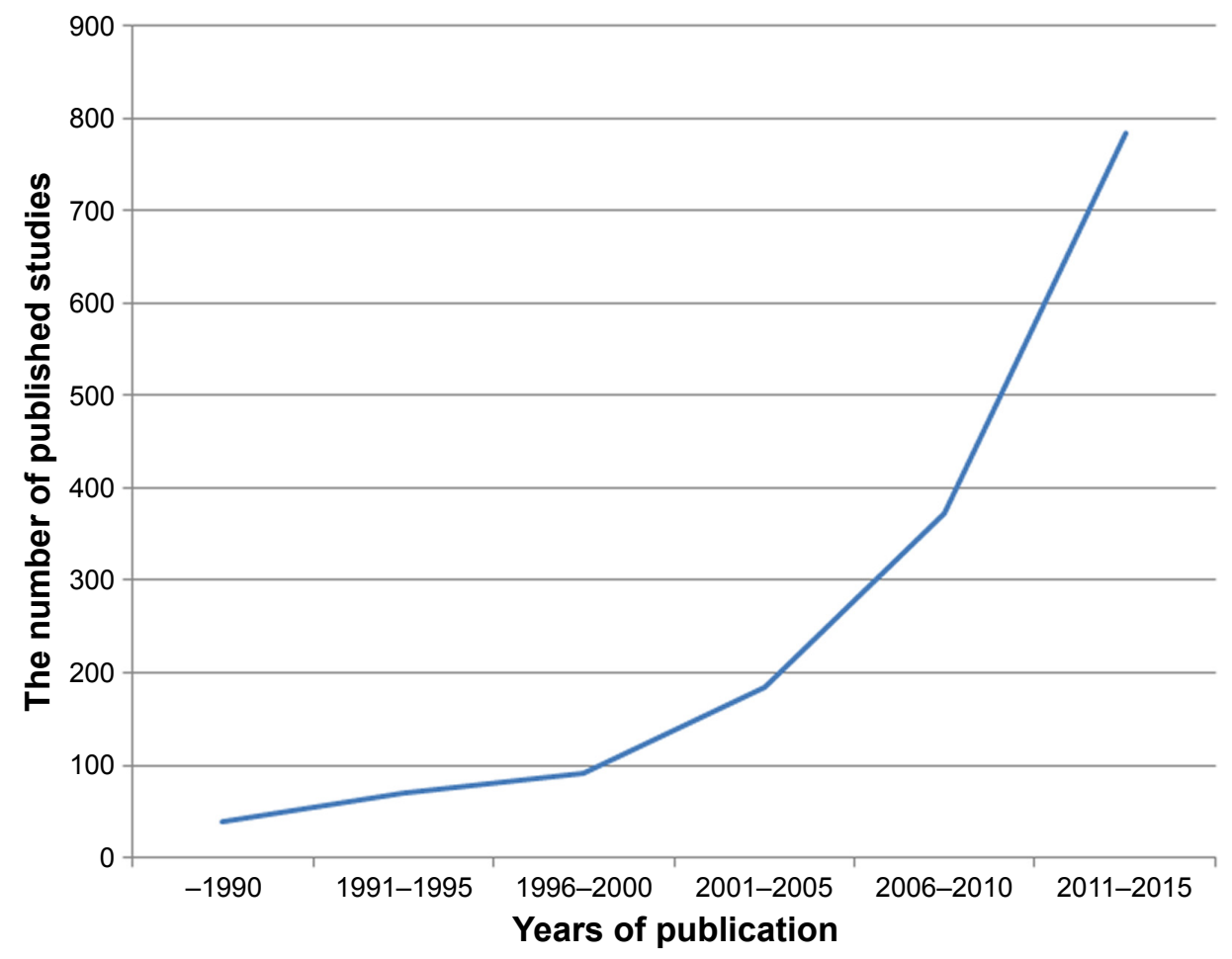

Figure I Numbers of published studies on radiotherapy for hepatocellular carcinoma over time. The counts were based on a search of the Scopus electronic database in March 2018 that was limited to original article. The search keywords included "hepatocellular carcinoma" AND "radiotherapy".

Liver cancer is a "silent killer" that is asymptomatic until significant disease progression. Therefore, it is not uncommon for patients to present with large tumors or major vascular invasion at diagnosis. The incidence of $\mathrm{HCC}$ is highest in East Asia, where screening is less popular and social awareness remains insufficient; therefore, physicians more often encounter advanced HCC patients in clinic. External beam radiation therapy (EBRT) has been increasingly used as a local treatment option for advanced HCCs, ${ }^{6,7}$ as threedimensional conformal radiotherapy (3DCRT) has enabled selective irradiation of tumors while sparing a significant volume of normal tissue.

Studies on radiation therapy for HCC have been rapidly increasing in recent years, ${ }^{8}$ and a number of important studies have been published recently. In response to this trend, the updated National Comprehensive Cancer Network (NCCN) guidelines increased the recommendation grade for radiation therapy for unresectable $\mathrm{HCC}$ from $2 \mathrm{~B}$ to $2 \mathrm{~A}$, the same grade as that for arterially directed therapy and ablation. ${ }^{9}$ Although EBRT has an emerging role as a local treatment for HCC, it is unfamiliar to some physicians treating HCC; possibly due to its different mechanism from those of conventional medical treatments, with its radiobiological and physical perspectives.

This review concisely presents the latest therapeutic results of EBRT for HCC so that all physicians treating HCC can assess the updated indications for use of EBRT for HCC. To better illustrate the current clinical results of EBRT for HCC, we mainly reviewed recently published meta-analyses or well-designed prospective studies. Studies written in languages other than English were not considered, and there were no restrictions regarding the publication years because the majority of clinical studies of RT for HCC have been published recently (Figure 1). We reviewed ongoing trials that can extend the indications for use of EBRT and reinforce the evidence and finally discuss the recommendation for EBRT based on a review of the current clinical practice guidelines.

\section{Discussion}

\section{History and indication of RT for HCC}

This section presents the main indications of radiotherapy (RT) for HCC for intrahepatic disease rather than extrahepatic metastases, according to historical trends.

In the era of two-dimensional RT, targeting tumors while sparing normal liver tissue was difficult to achieve. The whole-liver tolerance radiation dose with a $5 \%$ risk of radiation-induced liver disease is $30-35 \mathrm{~Gy},{ }^{10}$ which is not high enough to control HCCs. Therefore, RT for HCC was considered ineffective and HCC was erroneously assumed to be a radio-resistant tumor. ${ }^{11}$

3DCRT is based on RT treatment planning using 3D images obtained by computed tomography, which allows for 
targeting of liver tumors specifically while sparing normal liver tissue. In the 1990s, pioneering radiation oncologists treated intrahepatic tumors using 3DCRT with radiation doses of 45-60 Gy, with response rates ranging from $45 \%$ to $63 \%$, with acceptable toxicities. ${ }^{12-14}$

Intensity modulated RT, a more advanced EBRT technique after 3DCRT, uses modulated beams to optimize the dose distribution for tumors with complex shapes and to maximize the sparing of normal tissues through a computerized planning process. Its utilization has been increasing with its potency and increasing treatment efficacy while minimizing side effects. ${ }^{15,16}$ Stereotactic body radiotherapy (SBRT) is as an EBRT method that accurately delivers a high dose of irradiation in one or few fractions to extracranial target. ${ }^{17}$ SBRT has been increasingly used as a possible curative option for tumors that are unable to undergo conventional local treatment such as surgery or radiofrequency ablation (RFA). ${ }^{18}$

\section{Major vascular invasion}

Although 3DCRT enabled selective tumor irradiation while normal liver tissue could be spared, RT was not considered a primary option in the early days; it was mainly applied to patients with intractable disease with no other treatment options. Major vascular invasions such as portal vein thrombosis (PVT) are therapeutic challenges predicted to confer a poor prognosis. The median survival period of patients with PVT was less than 3 months without treatment. ${ }^{19,20}$

Sorafenib is the current treatment recommendation as per BCLC guidelines for these patients. In two landmark trials (SHARP ${ }^{21}$ and the Asia-Pacific study ${ }^{22}$ ), the sorafenib arms included $36 \%$ of macrovascular invasion cases. In the Asia-Pacific study, patients with macroscopic vascular invasion showed a survival benefit due to sorafenib in subgroup analysis (hazard ratio: $0.63,95 \%$ confidence interval [CI]: 0.39-1.03). The tumor response rates were moderate, which were $2 \%$ and $3.3 \%$ for all included patients in the SHARP and the Asia-Pacific study. A recent Korean study of 30 patients with main or first branch PVT reported a response rate of $10 \%$ and a median overall survival (OS) of 3.1 months (95\% CI: 2.7-3.5) with sorafenib monotherapy. ${ }^{23}$

The portal vein is a major vessel with abundant blood flow; thus, surgical approaches are challenging owing to the risk of bleeding. The complex anatomical structures related to the hepatic artery and bile duct are additional hindrances to the ablative approach. EBRT can be performed regardless of tumor location, and since radiation tolerance of the great vessels is $>100$ Gy in equivalent dose for 2 Gy per fraction treatment, as extrapolated from SBRT series of central lung cancers, ${ }^{24-26}$ PVT is a possible indication for EBRT. A propensity score analysis showed that EBRT can provide better survival (median survival period: 10.9 vs 4.8 months; $p=0.025$ ) than that of sorafenib alone in patients with PVT. ${ }^{27}$ In a meta-analysis by Rim et $\mathrm{al}^{28}$ of 22 observational studies and 1,903 patients, the pooled response rate of 3DCRT to HCC with PVT was 51.3\% (95\% CI: 45.7-57.0) among 18 studies, while the 1- and 2-year OS rates were $43.8 \%$ (95\% CI: 37.6-50.2) and 22.3\% (95\% CI: 17.7-27.6) among 22 and 18 studies, respectively. The toxicity was moderate, with $<10 \%$ of grade $\geq 3$ toxicity in the majority of the included studies; the most common grade $\geq 3$ toxicity was lymphocytopenia.

Inferior vena cava (IVC) invasion is another type of major vascular invasion, with a much rarer incidence rate of about $4 \%$ at diagnosis or during chemoembolization sessions of HCCs. ${ }^{29}$ Despite its rarity, IVC invasion may lead to serious complications such as pulmonary embolism, lung metastasis, heart failure, and may cause sudden death. ${ }^{30}$ In a recent meta-analysis including a total of 164 HCC patients with IVC invasion from eight observational studies, ${ }^{31}$ the pooled response rate was $59.2 \%$ (95\% CI: $39.0 \%-76.7 \%$ ) and the 1- and 2-year OS rates were $53.6 \%$ (95\% CI: 45.7\%-61.3\%) and 36.9\% (95\% CI: 29.8\%$44.8 \%$ ), respectively. One study reported one instance each of pulmonary embolism and esophageal rupture (primary toxicities of concern in HCC with IVC invasion), while no other grade $\geq 3$ toxicity was reported among the included studies. Thus, the overall grade $\geq 3$ toxicity rate was $1.2 \%$ (two of 164 patients).

For major vascular invasion, in which it might be difficult to perform conventional local treatments including surgery or RFA, EBRT has accumulated clinical experience showing it to be an efficient and feasible local treatment option.

\section{Combined RT as an option for unresectable HCCs}

$\mathrm{HCC}$ is a cancer in which various treatment modalities are applied. Transarterial chemoembolization (TACE) is the one of the most commonly used treatments for HCC. Although it is widely used, complete response is rarely observed with large tumors and there is a risk of hepatic failure in cases with PVT. ${ }^{32,33}$ RT has several advantages when performed after TACE. RT may elicit further responses in tumors that have shown partial responses to TACE. TACE increases the efficiency of RT by enhancing radiosensitivity and lipiodol uptake after TACE may be used as a tumor-targeting guide to enable more precise RT. ${ }^{34}$ Many researchers have compared the efficacy of TACE alone and in combination with RT. Huo and Eslick ${ }^{35}$ conducted a meta-analysis including 25 studies 
and 2,577 patients (including 11 randomized controlled trials [RCTs]) in which the TACE plus RT group showed better survival and tumor responses rate than those in the TACE-alone group (median OS: 22.7 vs 13.5 months; 1-year survival: odds ratio [OR] 1.36, 95\% CI: 1.19-1.54; 2-year survival: OR: 1.55, 95\% CI: 1.31-1.85; complete response: OR: $2.73,95 \%$ CI: 1.95-3.81).

Hepatic arterial infusion chemotherapy (HAIC) is less commonly used than TACE, and recent studies have mainly focused on the treatment of locally advanced or intractable tumors. Sumie et al compared TACE and HAIC with cisplatin (10 mg/person on day 1-5) and subsequent 5-fluorouracil (5-FU, $250 \mathrm{mg} /$ person on days 1-5) regimen. ${ }^{36}$ The tumor response rate was higher in the HAIC group, and the HAIC group showed survival benefit in tumors $>5 \mathrm{~cm}$ in diameter. The HCC multidisciplinary team at the Yonsei Cancer Center reported the down-staging effects of combining HAIC and EBRT. ${ }^{37}$ Among the included patients with unresectable tumors, $16.9 \%$ underwent curative resection after down staging, with a 5-year survival rate of $49.6 \%$. An updated report from the same center analyzed 637 BCLC stage C (BCLC-C) HCC patients who underwent HAIC and EBRT; ${ }^{38}$ the median OS of all patients was 15 months and reached 39 months in those who received $>60$ Gy of EBRT.

The combined treatment of TACE and RT is one of the most studied areas of RT indications and is expected to have an important role as a treatment option for locally advanced HCC. Previous studies have suggested that HAIC might be advantageous for advanced HCCs; combining HAIC and EBRT may provide a new possibility for cure but additional studies are warranted.

\section{SBRT}

SBRT is currently one of the most interesting treatment modalities in oncology since it can provide a curative effect similar to surgery or ablation, and, thus, provides an alternative treatment option in cases unsuitable for conventional modalities. SBRT delivers very high doses to tumors in a shorter time than does conventional RT. A short treatment period and a higher dose confer a higher cure rate but also result in a higher risk of side effects. SBRT utilizes advanced techniques such as cone beam image guidance, breath gating, and tumor tracking to offset the risk of side effects by maximizing the treatment precision. ${ }^{39-41}$

Recent prospective studies revealed a potential role for SBRT as a local ablative modality in HCC. Bujold et $\mathrm{al}^{42}$ performed SBRT in 102 advanced HCC cases (55\% cases with PVT) with a median target diameter of $7.2 \mathrm{~cm}$. The 1 -year local control rate was $87 \%$; the most common grade $\geq 3$ toxicity was liver related, including elevation of liver enzyme or bilirubin levels, cholangitis, and liver failure in $23.5 \%$ of patients. Lasley et al ${ }^{43}$ performed SBRT in $59 \mathrm{HCC}$ patients, including 21 Child-Pugh class (CPC) B patients (35.6\%) and 12 patients $(20.3 \%)$ with PVT. The 1 -year local control rates were $91 \%$ and $82 \%$ for CPC-A and CPC-B patients, respectively. Grade III or IV liver toxicity was observed in $38 \%$ of CPC-B patients. In a study by Scorsetti et al, ${ }^{44}$ a 1-year local control rate of $85.8 \%$ and a median survival period of 18 months were observed among 43 patients who underwent SBRT. Seven of 43 patients (16\%) experienced grade $\geq 3$ liver toxicity and five of the seven patients were CPC-B. Summarizing the prospective trials, the local control rates with SBRT were favorable, ranging from $86 \%$ to $95 \%$ in 1 year, ${ }^{42-45}$ however, possible liver toxicity should be cautiously considered, especially in patients with poor liver function.

In a large comparative meta-analysis by Qi et al including 70 observational studies and 5,204 patients, ${ }^{46}$ the SBRT group showed a pooled local control rate of $87 \%$ (95\% CI: 83-92) and a 1-year OS rate of 80\% (95\% CI: 71-87), which were not different from those of the charged particle therapy group. The acute grade $\geq 3$ pooled hepatic, bone marrow, and overall toxicity rates were 4.9\% (95\% CI: 3.0-8.1), 4.9\% (95\% CI: 3.4-7.2), and 9.6\% (95\% CI: 6.0-15.1), respectively. The pooled late toxicity rate was $6.4 \%(95 \%$ CI: $4.0 \%-10.1 \%$ ). A comparative meta-analysis of RT for HCC with PVT was performed, involving 37 observational studies comprising 2,513 patients. Three radiation therapy modalities were compared, and SBRT showed a favorable response rate of $70.7 \%$ (95\% CI: 63.7-76.8) as compared to that of 3DCRT (51.3\%, 95\% CI: 45.7-57.0) and selective internal radiation therapy $(33.3 \%, 95 \% \mathrm{CI}: 18.0-53.2) .{ }^{28}$ The severe toxicity rates were moderate, and less than $10 \%$ of grade $\geq 3$ toxicities were observed in most of the included studies. PVT generally occurs close to the duodenum, which is radiosensitive and is the dose-limiting organ for EBRT. Thus, applying high-dose RT such as SBRT is difficult. Nevertheless, considering the above studies, PVT may also be an indication for SBRT.

SBRT has advantages such as favorable tumor control, shorter duration than conventional RT, and a treatment less restricted by tumor location than other ablative treatments. Clinical and research interests in SBRT have been rapidly increasing and SBRT is expected to play an important role in the treatment of HCC.

Table 1 summarizes the referenced literature and up-todate reports. The representative studies and meta-analyses have been included to facilitate a quicker understanding of 


\begin{tabular}{|c|c|c|c|c|c|c|c|c|c|c|}
\hline \multicolumn{2}{|c|}{ 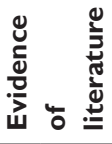 } & 离 & 3. & \multicolumn{2}{|l|}{ 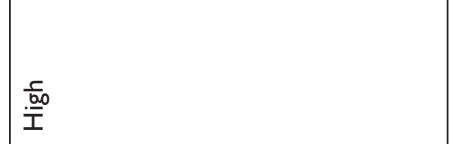 } & \multicolumn{2}{|l|}{ 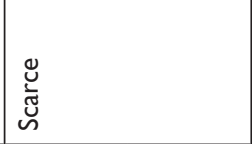 } & \multicolumn{3}{|l|}{ 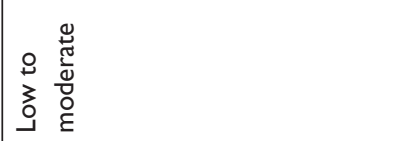 } \\
\hline \multicolumn{2}{|c|}{ 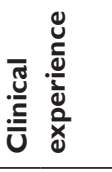 } & 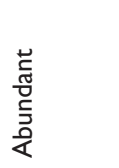 & 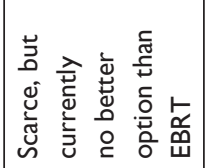 & \multicolumn{2}{|l|}{ 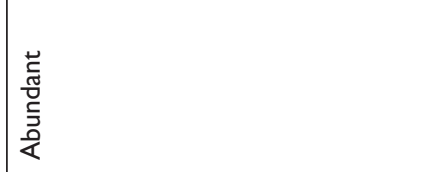 } & \multicolumn{2}{|l|}{ 峁 } & \multicolumn{3}{|l|}{ 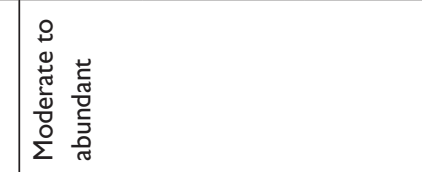 } \\
\hline \multicolumn{2}{|c|}{ 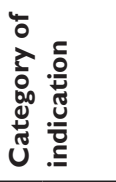 } & 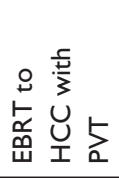 & 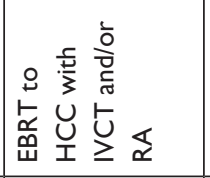 & \multicolumn{2}{|l|}{ 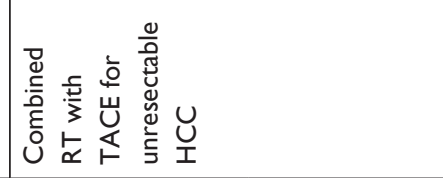 } & \multicolumn{2}{|c|}{ 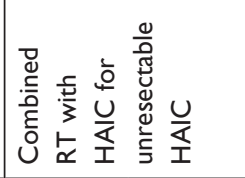 } & \multicolumn{3}{|c|}{ 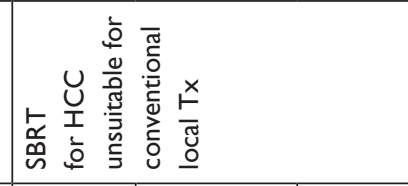 } \\
\hline \multicolumn{2}{|l|}{ 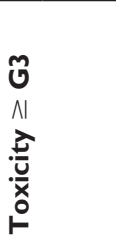 } & 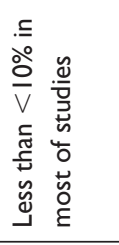 & 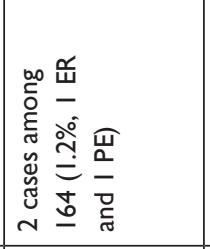 & 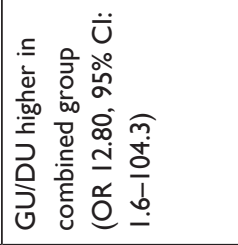 & & & 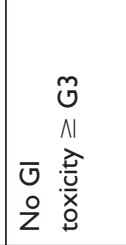 & 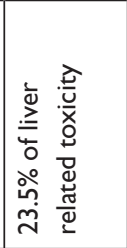 & 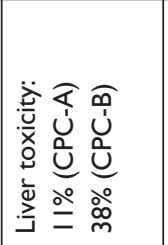 & 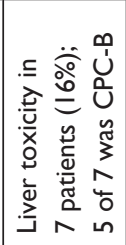 \\
\hline \multirow{3}{*}{ 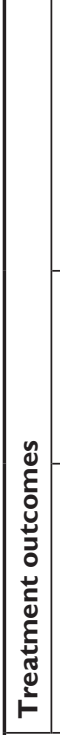 } & 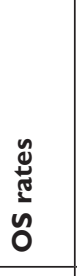 & 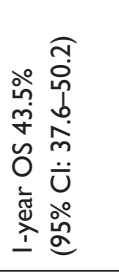 & 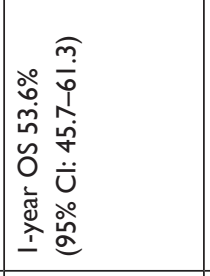 & 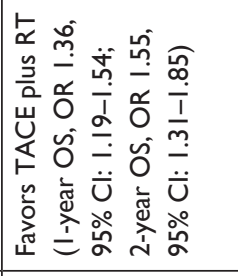 & \multirow{2}{*}{ 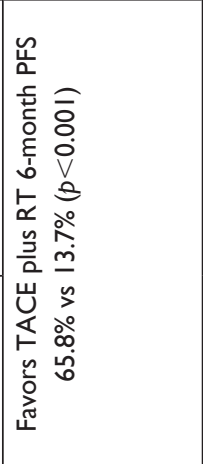 } & \multirow{2}{*}{ 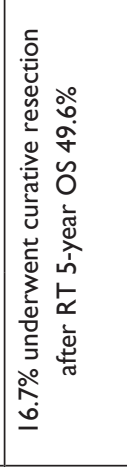 } & \multirow{2}{*}{ 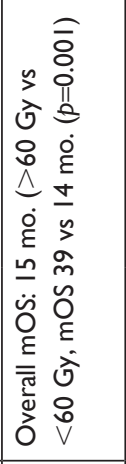 } & & 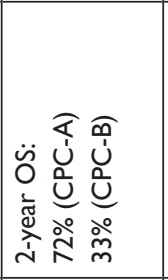 & \\
\hline & $\begin{array}{l}\text { on } \\
\frac{\pi}{\pi} \\
\frac{\pi}{\pi} \\
\Sigma\end{array}$ & 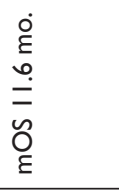 & 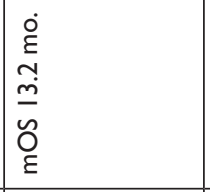 & 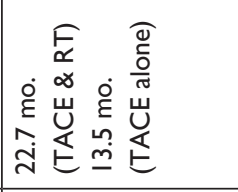 & & & & 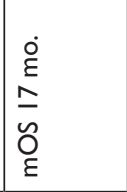 & 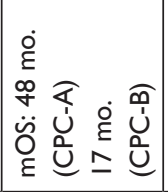 & $\begin{array}{c}\dot{d} \\
\dot{\infty} \\
\underline{\infty} \\
\ddot{\ddot{O}} \\
\dot{\varepsilon}\end{array}$ \\
\hline & 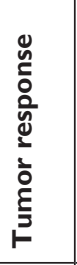 & 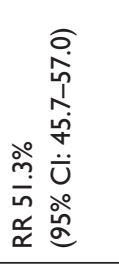 & 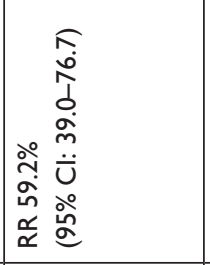 & 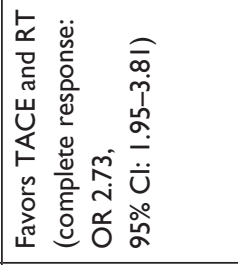 & 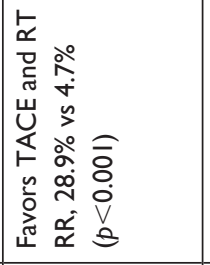 & & & 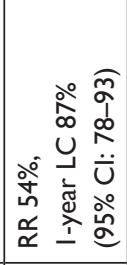 & 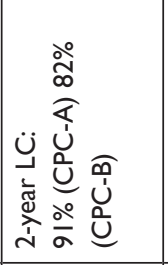 & 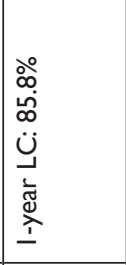 \\
\hline \multicolumn{2}{|c|}{ 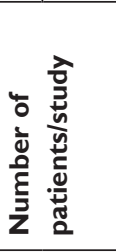 } & 旁 & 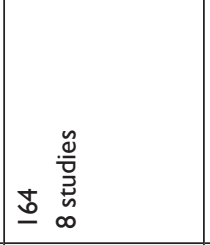 & 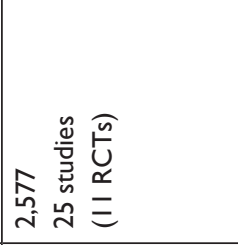 & 8 & $\stackrel{m}{\sim}$ & 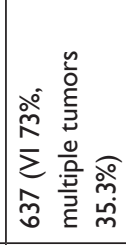 & 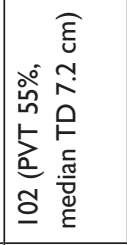 & 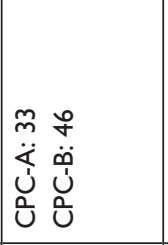 & 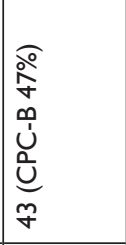 \\
\hline \multicolumn{2}{|c|}{ 突。 } & $\Sigma$ & $\Sigma$ & $\Sigma$ & 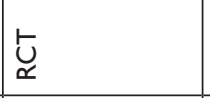 & $\mathscr{\not}$ & $\tilde{\alpha}$ & ๓ & 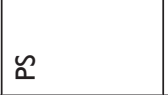 & $\check{\alpha}$ \\
\hline 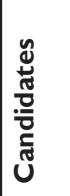 & & 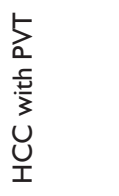 & 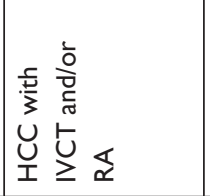 & 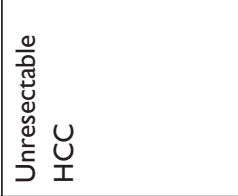 & 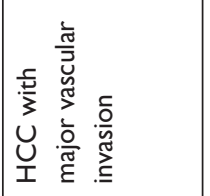 & 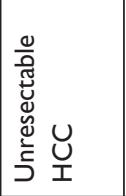 & 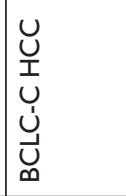 & 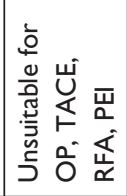 & $\underset{U}{u}$ & 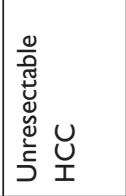 \\
\hline 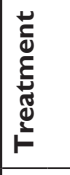 & & $\begin{array}{l}\text { 总 } \\
\text { ò } \\
\text { m }\end{array}$ & \begin{tabular}{|l} 
咅 \\
总
\end{tabular} & 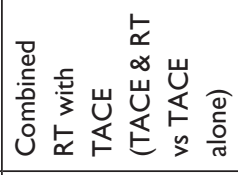 & 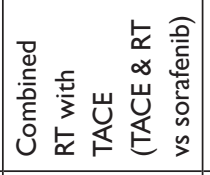 & 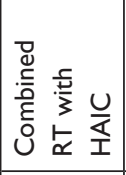 & 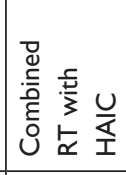 & 占 & $\begin{array}{l}\text { 品 } \\
\text { 心s }\end{array}$ & 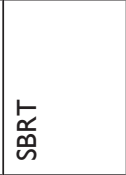 \\
\hline 辛 & & 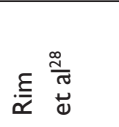 & 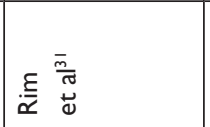 & 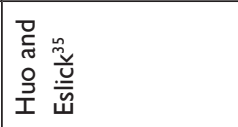 & 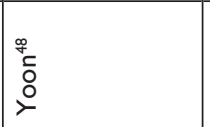 & 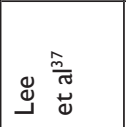 & 衰商 & 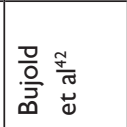 & 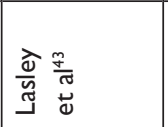 & 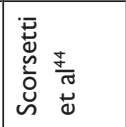 \\
\hline
\end{tabular}



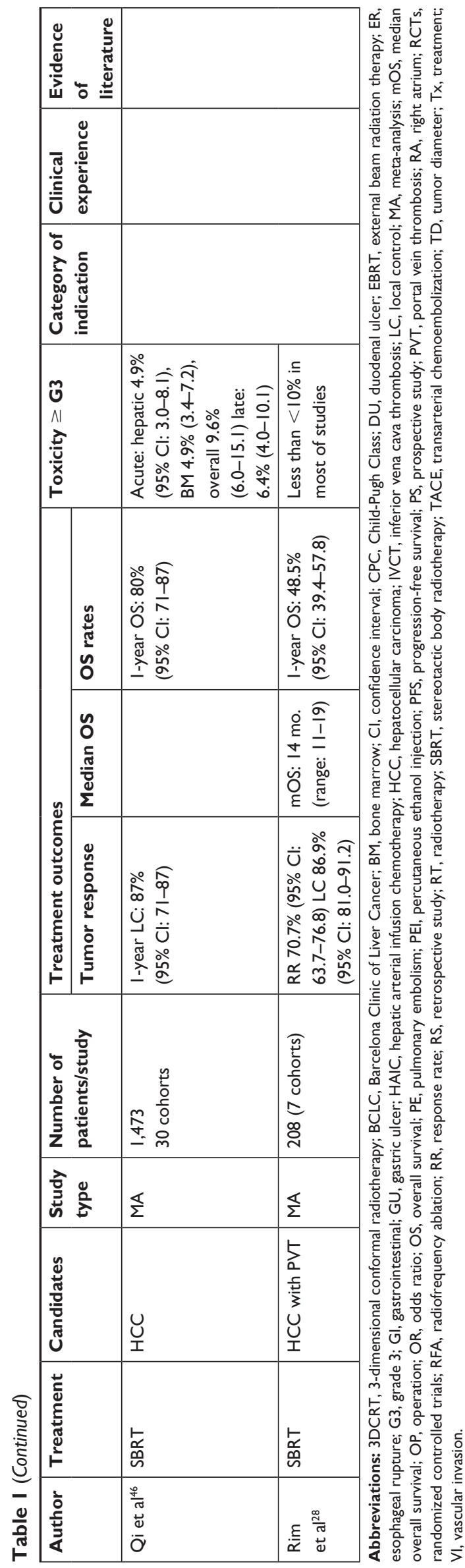

treatment modalities and outcomes. The studies have been classified into five categories of indications: (1) EBRT in HCC with PVT, (2) EBRT in HCC with IVC and/or right atrium invasion, (3) combined RT with TACE in unresectable HCC, (4) combined RT with HAIC in unresectable HCC, and (5) SBRT in HCC unsuitable for conventional local therapies. We have also included a recommendation for each indication based on relevant clinical experience and literature evidence. Clinical experience was evaluated considering the number of studies, patients, and period of history of the relevant indication (Table S1). Evidence in the literature was regarded as high if the evidence for the indication was obtained from RCTs or meta-analyses including RCTs. The evidence level was considered low if the evidence for the indication was mainly based on retrospective observational studies. Evidence gathered from prospective and controlled studies was considered a moderate level of evidence. When the number of studies was insufficient to make any recommendation, the evidence level was considered scarce.

\section{Up-to-date review of trials}

The number of clinical trials of EBRT for HCC is increasing, potentially improving the future grade of evidence. In this section, we briefly review the trials registered at ClinicalTrials.gov, which may potentially extend the EBRT indication.

We used the search term: "liver cancer AND radiotherapy AND randomized NOT yttrium." This term was designed to selectively identify RCTs in order to find potential candidates with high-grade evidence of EBRT efficacy. Internal RT using yttrium was not a subject of concern in this review.

Eighty-three studies were found initially, of which 54 were excluded from review because (1) the target disease was not HCC (eg, liver metastasis, cholangiocarcinoma, or including all gastrointestinal cancers), (2) the treatment modality of subject was not EBRT, or (3) no comparative control group was included.

The remaining 29 studies were RCTs evaluating EBRT for HCC: $11(37.9 \%)$ by a primary institution in the USA, four in China (13.8\%), three in Canada (10.3\%), three in Korea $(10.9 \%)$, two in Belgium, two in India, two in Taiwan, and one in France. Eight studies were about TACE versus TACE with EBRT, which is one of the most commonly used combined treatments using EBRT. Although combined treatment showed a survival benefit in meta-analysis (including 17 trials and 1,475 patients in five RCTs; 1-, 2-, and 3-year OS: OR: 2.23, 95\% CI: 1.76-2.83; OR: $2.39,95 \%$ CI: $1.85-3.09$; and OR: $2.75,95 \%$ CI: $2.08-3.64$, respectively), ${ }^{47}$ the researchers 
are trying to consolidate its benefit and upgrade the evidence with more well-designed RCTs.

Of the remaining 21 studies, two studies of TACE versus SBRT using a CyberKnife for recurrent HCC had a withdrawn status. Of the 19 studies remaining, two were incomplete, 16 were still recruiting, and one had an unknown status. Of 16 recruiting studies, eight (50\%) were comparing TACE and EBRT (including SBRT). Two studies (12.5\%) were comparing RFA and proton therapy as local ablative modalities. The efficacy of EBRT added to sorafenib therapy was assessed in two studies (12.5\%). Table 2 summarizes these 19 studies, which may further extend the indication of EBRT.

Of two completed studies, one (NCT02724475, China) compared laser ablation and EBRT for intermediate and advanced HCC, although the results were not available. The second study (NCT01901692, Korea) compared sorafenib with TACE and EBRT for HCC with major vascular invasion. A total of 90 patients were randomized; the progressionfree survival rates, which were the primary endpoints of the study, were $13.7 \%$ and $65.8 \%$ at 6 months in the sorafenib and TACE and EBRT arms, respectively $(p<0.001)$. The response rates were $4.7 \%$ and $28.9 \%$ in the sorafenib and TACE and EBRT arms, respectively $(p<0.001)$. Most adverse events were grade 1 or 2 in both arms. ${ }^{48}$

In summary, the pending results of the NCT01901692 study may expand the indications for use of EBRT application; combined TACE and EBRT may possibly be indicated for HCC with major vascular invasion, which is currently categorized as BCLC-C, with sorafenib as the recommended treatment. Further expected results include a comparison of TACE and EBRT, a comparison of EBRT with RFA as an ablative option, and a combination of local EBRT with systemic sorafenib treatment.

\section{Evidence grading of EBRT for $\mathrm{HCC}$ in guidelines}

Although EBRT for HCC is commonly performed in clinical practice, its evidence has been underestimated in some international guidelines.

One of the most widely used and clinically cited guidelines is that from the European Association for the Study of the Liver-European Organization for Research and Treatment of Cancer (EASL-EORTC). ${ }^{5}$ The EASL-EORTC guidelines have much in common with those of the BCLC, such as recommending sorafenib as a single standard for patients with advanced-stage disease (including distant and lymph node metastasis and vascular invasion). EBRT was evaluated to have no scientific evidence and no indication other than palliation of bone metastases, as described in the guidelines. Although the guidelines published by American Association for the Study of Liver Disease (AASLD) cited recent studies on EBRT, there is little mention of the role of EBRT and systemic therapy over no therapy is recommended for patients with macrovascular invasion and/or metastasis. ${ }^{49}$

Table 2 Ongoing randomized controlled trials of EBRT for HCC

\begin{tabular}{|c|c|c|c|c|c|}
\hline $\begin{array}{l}\text { Trial } \\
\text { number }\end{array}$ & $\begin{array}{l}\text { Country of } \\
\text { primary facility }\end{array}$ & Control arm & EBRT arm & Target disease & $\begin{array}{l}\text { Current } \\
\text { status }\end{array}$ \\
\hline NCT0I90I692 & Korea & Sorafenib & TACE and EBRT & HCC with major vascular invasion & Completed \\
\hline NCT02724475 & China & Laser & 3DCRT & Intermediate and advanced HCC & Completed \\
\hline NCT02470533 & Belgium & TACE & SBRT & $\mathrm{HCC}(\mathrm{BCLC}$ A-B) & Recruiting \\
\hline NCT02I 25396 & China & TACE & EBRT & Postoperative HCC & Recruiting \\
\hline NCT03338647 & India & TACE & SBRT & Advanced HCC & Recruiting \\
\hline NCT00857805 & USA & TACE & Proton & HCC not exceeding San Francisco criteria & Recruiting \\
\hline NCT02I 82687 & USA & TACE & SBRT & HCC within Milan criteria (bridging therapy) & Recruiting \\
\hline NCT02323360 & Italy & re-TACE & SBRT & Incomplete TACE of unresectable HCC & Recruiting \\
\hline NCT03326375 & Korea & re-TACE & SBRT & Incomplete TACE of HCC & Recruiting \\
\hline NCT0292I I39 & Taiwan & re-TACE & SBRT & HCC with incomplete response of TACE & Recruiting \\
\hline NCTOI I 4 I 478 & USA & Sorafenib & Sorafenib and proton RT & HCC exceeding San Francisco criteria & Recruiting \\
\hline NCT0I 730937 & USA & Sorafenib & Sorafenib and SBRT & Advanced, recurrent HCC & Recruiting \\
\hline NCT0I963429 & Korea & RFA & Proton & Recurrent or residual small HCC & Recruiting \\
\hline NCT02640924 & Taiwan & RFA & Proton & Medium or large HCC & Recruiting \\
\hline NCT03I 72559 & Canada & None & SBRT & $\mathrm{HCC}$ ineligible to TACE & Recruiting \\
\hline NCT025II 522 & Canada & Supportive care & Palliative EBRT & HCC or liver metastases & Recruiting \\
\hline NCT03।68I52 & USA & Microwave ablation & SBRT & Localized HCC & Recruiting \\
\hline NCT02239900 & USA & Ipilimumab & SBRT & Advanced solid tumors (liver and lung) & Recruiting \\
\hline NCT00557024 & China & RFA & RFA and RT & HCC performed prior RFA & Unknown \\
\hline
\end{tabular}

Abbreviations: BCLC, Barcelona Clinic of Liver Cancer; EBRT, external beam radiation therapy; HCC, hepatocellular carcinoma; TACE, transarterial chemoembolization; 3DCRT, 3-dimensional conformal radiotherapy; SBRT, stereotactic body radiotherapy; RFA, radiofrequency ablation; RT, radiotherapy. 
On the other hand, Asian guidelines describe some roles of EBRT for HCC. The Asia-Pacific Primary Liver Cancer Expert Meeting guidelines provide indications for use of EBRT for each stage of the BCLC system and provide evidence levels for several indications, including SBRT for RFA-refractory HCC and combined EBRT and TACE for locally advanced HCC. ${ }^{50}$ The guidelines from the Korean Liver Cancer Society classify the clinical status of HCC anatomically according to modified Union for International Cancer Control staging and describe the best and alternative options. ${ }^{51}$ The provision of multiple options might be useful in determining the treatment modality in clinical practice, as there may be a variety of disease statuses in practice, even if they are included in one stage in a given staging system.

EBRT for HCC is rapidly increasing in terms of treatment experience and the number of studies. Encouragingly, to reflect this trend, the NCCN guidelines updated in 2018 have elevated the evidence level of EBRT as a local treatment for unresectable $\mathrm{HCC}$, from $2 \mathrm{~B}$ to $2 \mathrm{~A}$, which is same grade as that for arterially directed therapy and ablation. ${ }^{9}$ In addition, the recommendation was strengthened by eliminating uncertain words such as "suggest" and "possible" for the efficacy and safety of EBRT.

In the EASL-EORTC and AASLD guidelines, the lack of randomized trials was the main reason to not acknowledge the efficacy of EBRT. However, a randomized trial of sorafenib versus combined TACE and EBRT (ClinicalTrials. gov trial number NCT01901692) is pending publication; the difference in treatment outcomes between the two groups was apparent in the abstract. ${ }^{48}$ In addition, the latest EASL guidelines were published in 2011. Since then, much more research on EBRT for HCC has been published than that available before 2011 (Figure 1). Hence an updated assessment of the accumulated clinical experience is also expected.

\section{Conclusion}

This review provides a practical introduction to the outcomes and indications pertaining to EBRT for physicians treating HCC. We recommend Table 1 as a quick reference to aid in decision making in multidisciplinary meetings. We expect the upcoming results of well-designed trials to consolidate the efficacy of EBRT in the near future.

\section{Disclosure}

The authors report no conflicts of interest in this work.

\section{References}

1. Torre LA, Bray F, Siegel RL, Ferlay J, Lortet-Tieulent J, Jemal A. Global cancer statistics, 2012. CA Cancer J Clin. 2015;65(2):87-108.
2. Beasley RP, Hwang LY, Lin CC, Chien CS. Hepatocellular carcinoma and hepatitis B virus: a prospective study of 22,707 men in Taiwan. Lancet. 1981;2(8256):1129-1133.

3. Sakuma K, Saitoh N, Kasai M, et al. Relative risks of death due to liver disease among Japanese male adults having various statuses for hepatitis B s and e antigen/antibody in serum: a prospective study. Hepatology. 1988;8(6):1642-1646.

4. Llovet JM, Bru C, Bruix J. Prognosis of hepatocellular carcinoma: the BCLC staging classification. Semin Liver Dis. 1999;19(3):329-338.

5. European Association for the Study of the Liver; European Organisation for Research and Treatment of Cancer. EASL-EORTC clinical practice guidelines: management of hepatocellular carcinoma. $J$ Hepatol. 2012;56(4):908-943.

6. Cha H, Park HC, Yu JI, et al. Clinical practice patterns of radiotherapy in patients with hepatocellular carcinoma: a Korean Radiation Oncology Group Study (KROG 14-07). Cancer Res Treat. 2017;49(1):61-69.

7. Cheng S, Chen M, Cai J. Chinese expert consensus on multidisciplinary diagnosis and treatment of hepatocellular carcinoma with portal vein tumor thrombus: 2016 edition. Oncotarget. 2017;8(5):8867-8876.

8. Rim CH, Seong J. Application of radiotherapy for hepatocellular carcinoma in current clinical practice guidelines. Radiat Oncol J. 2016;34(3):160-167.

9. National Comprehensive Cancer Network (NCCN) ${ }^{\circledR}$. NCCN Guidelines version 1. 2018, hepatobiliary cancers. Available from: https://www. nccn.org/professionals/physician_gls/pdf/hepatobiliary.pdf. Accessed April 2, 2018.

10. Emami B, Lyman J, Brown A, et al. Tolerance of normal tissue to therapeutic irradiation. Int J Radiat Oncol Biol Phys. 1991;21(1):109-122.

11. Feng M, Ben-Josef E. Radiation therapy for hepatocellular carcinoma. Semin Radiat Oncol. 2011;21(4):271-277.

12. Ben-Josef E, Normolle D, Ensminger WD, et al. Phase II trial of high-dose conformal radiation therapy with concurrent hepatic artery floxuridine for unresectable intrahepatic malignancies. J Clin Oncol. 2005;23(24):8739-8747.

13. Dawson LA, McGinn CJ, Normolle D, et al. Escalated focal liver radiation and concurrent hepatic artery fluorodeoxyuridine for unresectable intrahepatic malignancies. J Clin Oncol. 2000;18(11):2210-2118.

14. Seong J, Keum KC, Han KH, et al. Combined transcatheter arterial chemoembolization and local radiotherapy of unresectable hepatocellular carcinoma. Int J Radiat Oncol Biol Phys. 1999;43(2):393-397.

15. Kim TH, Park JW, Kim YJ, et al. Simultaneous integrated boostintensity modulated radiation therapy for inoperable hepatocellular carcinoma. Strahlenther Onkol. 2014;190(10):882-890.

16. Citrin DE. Recent developments in radiotherapy. N Engl J Med. 2017; 377(22):2200-2201.

17. Guckenberger M, Andratschke N, Alheit H, et al; Deutschen Gesellschaft für Radioonkologie (DEGRO). Definition of stereotactic body radiotherapy: principles and practice for the treatment of stage I nonsmall cell lung cancer. Strahlenther Onkol. 2014;190(1):26-33.

18. Sanuki N, Takeda A, Kunieda E. Role of stereotactic body radiation therapy for hepatocellular carcinoma. World J Gastroenterol. 2014;20(12): 3100-3111.

19. Llovet JM, Bustamante J, Castells A, et al. Natural history of untreated nonsurgical hepatocellular carcinoma: rationale for the design and evaluation of therapeutic trials. Hepatology. 1999;29(1):62-67.

20. Nagasue N, Yukaya H, Hamada T, Hirose S, Kanashima R, Inokuchi K. The natural history of hepatocellular carcinoma. A study of 100 untreated cases. Cancer. 1984;54(7):1461-1465.

21. Llovet JM, Ricci S, Mazzaferro V, et al; SHARP Investigators Study Group. Sorafenib in advanced hepatocellular carcinoma. NEngl J Med. 2008;359(4):378-390.

22. Cheng AL, Kang YK, Chen Z, et al. Efficacy and safety of sorafenib in patients in the Asia-Pacific region with advanced hepatocellular carcinoma: a phase III randomised, double-blind, placebo-controlled trial. Lancet Oncol. 2009;10(1):25-34.

23. Jeong SW, Jang JY, Shim KY, et al. Practical effect of sorafenib monotherapy on advanced hepatocellular carcinoma and portal vein tumor thrombosis. Gut Liver. 2013;7(6):696-703. 
24. Bezjak A, Paulus R, Gaspar L, et al. Primary study endpoint analysis for NRG oncology/RTOG 0813 trial of stereotactic body radiation therapy (SBRT) for centrally located non-small cell lung cancer (NSCLC). Int J Radiat Oncol Biol Phys. 2016;94(1):5-6.

25. National Comprehensive Cancer Network $(\mathrm{NCCN})^{\circledR}$. Principle of radiation therapy. NCCN guidelines version 4.2018, non-small cell lung cancer. Available from: https://www.nccn.org/professionals/physician gls/pdf/nscl.pdf. Assessed April 2, 2018.

26. Videtic GM, Hu C, Singh AK, et al. A randomized phase 2 study comparing 2 stereotactic body radiation therapy schedules for medically inoperable patients with stage I peripheral non-small cell lung cancer: NRG oncology RTOG 0915 (NCCTG N0927). Int J Radiat Oncol Biol Phys. 2015;93(4):757-764.

27. Nakazawa T, Hidaka H, Shibuya A, et al. Overall survival in response to sorafenib versus radiotherapy in unresectable hepatocellular carcinoma with major portal vein tumor thrombosis: propensity score analysis. BMC Gastroenterol. 2014;14:84.

28. Rim CH, Kim CY, Yang DS, Yoon WS. Comparison of radiation therapy modalities for hepatocellular carcinoma with portal vein thrombosis: a meta-analysis and systematic review. Radiother Oncol. Epub 2017 Dec 9.

29. Lee IJ, Chung JW, Kim HC, et al. Extrahepatic collateral artery supply to the tumor thrombi of hepatocellular carcinoma invading inferior vena cava: the prevalence and determinant factors. J Vasc Interv Radiol. 2009;20(1):22-29.

30. Koo JE, Kim JH, Lim YS, et al. Combination of transarterial chemoembolization and three-dimensional conformal radiotherapy for hepatocellular carcinoma with inferior vena cava tumor thrombus. Int J Radiat Oncol Biol Phys. 2010;78(1):180-187.

31. Rim CH, Kim CY, Yang DS, Yoon WS. External beam radiation therapy to hepatocellular carcinoma involving inferior vena cava and/or right atrium: a meta-analysis and systemic review. Radiother Oncol. Epub 2018 Mar 29.

32. Ryder SD, Rizzi PM, Metivier E, Karani J, Williams R. Chemoembolisation with lipiodol and doxorubicin: applicability in British patients with hepatocellular carcinoma. Gut. 1996;38(1):125-128.

33. Ebied OM, Federle MP, Carr BI, et al. Evaluation of responses to chemoembolization in patients with unresectable hepatocellular carcinoma. Cancer. 2003;97(4):1042-1050.

34. Lee IJ, Seong J. Radiosensitizers in hepatocellular carcinoma. Semin Radiat Oncol. 2011;21(4):303-311.

35. Huo YR, Eslick GD. Transcatheter arterial chemoembolization plus radiotherapy compared with chemoembolization alone for hepatocellular carcinoma: a systematic review and meta-analysis. JAMA Oncol. 2015;1(6):756-765.

36. Sumie S, Yamashita F, Ando E, et al. Interventional radiology for advanced hepatocellular carcinoma: comparison of hepatic artery infusion chemotherapy and transcatheter arterial lipiodol chemoembolization. AJR Am J Roentgenol. 2003;181(5):1327-1334.

37. Lee HS, Choi GH, Choi JS, et al. Surgical resection after down-staging of locally advanced hepatocellular carcinoma by localized concurrent chemoradiotherapy. Ann Surg Oncol. 2014;21(11):3646-3653.

38. Byun HK, Kim HJ, Im YR, Seong J. Liver-directed concurrent chemoradiotherapy with high-dose radiation is effective in Barcelona Clinic Liver Cancer stage $\mathrm{C}$ hepatocellular carcinoma. Poster presented at: The 35th Annual Meeting of Korean Society for Radiation Oncology; October 13; 2017; Seoul, Korea: S133.
39. Guckenberger M, Sweeney RA, Wilbert J, et al. Image-guided radiotherapy for liver cancer using respiratory-correlated computed tomography and cone-beam computed tomography. Int J Radiat Oncol Biol Phys. 2008;71(1):297-304.

40. Guckenberger M, Meyer J, Wilbert J, et al. Intra-fractional uncertainties in cone-beam CT based image-guided radiotherapy (IGRT) of pulmonary tumors. Radiother Oncol. 2007;83(1):57-64.

41. Kubo HD, Hill BC. Respiration gated radiotherapy treatment: a technical study. Phys Med Biol. 1996;41(1):83-91.

42. Bujold A, Massey CA, Kim JJ, et al. Sequential phase I and II trials of stereotactic body radiotherapy for locally advanced hepatocellular carcinoma. J Clin Oncol. 2013;31(13):1631-1639.

43. Lasley FD, Mannina EM, Johnson CS, et al. Treatment variables related to liver toxicity in patients with hepatocellular carcinoma, Child-Pugh class A and B enrolled in a phase 1-2 trial of stereotactic body radiation therapy. Pract Radiat Oncol. 2015;5(5):e443-e449.

44. Scorsetti M, Comito T, Campisi M, et al. The challenge of inoperable hepatocellular carcinoma (HCC): results of a single-institutional experience on stereotactic body radiation therapy (SBRT). Int J Radiat Oncol Biol Phys. 2014;90(1):S376-S377.

45. Kang JK, Kim MS, Cho CK, et al. Stereotactic body radiation therapy for inoperable hepatocellular carcinoma as a local salvage treatment after incomplete transarterial chemoembolization. Cancer. 2012;118(21):5424-5431.

46. Qi WX, Fu S, Zhang Q, Guo XM. Charged particle therapy versus photon therapy for patients with hepatocellular carcinoma: a systematic review and meta-analysis. Radiother Oncol. 2015;114(3):289-295.

47. Meng MB, Cui YL, Lu Y, et al. Transcatheter arterial chemoembolization in combination with radiotherapy for unresectable hepatocellular carcinoma: a systematic review and meta-analysis. Radiother Oncol. 2009;92(2):184-194.

48. Yoon SM. Sorafenib versus transarterial chemoembolization plus external beam radiotherapy in patients with hepatocellular carcinoma with major vascular invasion (START): a randomized phase 2 trial. Washington, DC: The Liver Meeting; 2017. Available from: http:// liverlearning.aasld.org/aasld/2017/thelivermeeting/194907/sang.min. yoon.sorafenib.versus.transarterial.chemoembolization.plus.external. html. Accessed November 20, 2017.

49. Heimbach JK, Kulik LM, Finn RS, et al. AASLD guidelines for the treatment of hepatocellular carcinoma. Hepatology. 2018;67(1):358-380.

50. Park HC, Yu JI, Cheng JC, et al. Consensus for radiotherapy in hepatocellular carcinoma from the 5th Asia-Pacific Primary Liver Cancer Expert Meeting (APPLE 2014): current practice and future clinical trials. Liver Cancer. 2016;5(3):162-174.

51. Korean Liver Cancer Study Group (KLCSG); National Cancer Center, Korea (NCC). 2014 Korean Liver Cancer Study Group-National Cancer Center Korea practice guideline for the management of hepatocellular carcinoma. Korean J Radiol. 2015;16(3):465-522. 


\section{Supplementary material}

Table SI Search keywords and numbers of published studies relevant to the indications for external beam radiation therapy (EBRT) for hepatocellular carcinoma (HCC). Publication counts are based on a search limited to original articles in the Scopus database performed in March 2018

\begin{tabular}{|c|c|c|c|}
\hline Category of indication & Search keywords & $\begin{array}{l}\text { Number of } \\
\text { published } \\
\text { clinical articles }\end{array}$ & $\begin{array}{l}\text { Year with } \geq 5 \text { studies } \\
\text { published annually }\end{array}$ \\
\hline $\begin{array}{l}\text { EBRT to } \mathrm{HCC} \text { with portal vein } \\
\text { thrombosis (PVT) }\end{array}$ & $\begin{array}{l}\text { (radiotherapy OR "radiation therapy") AND } \\
\text { ("hepatocellular carcinoma" OR HCC) } \\
\text { AND ("portal vein thrombosis" OR PVT OR PVTT) }\end{array}$ & 217 & 2007 \\
\hline $\begin{array}{l}\text { EBRT to } \mathrm{HCC} \text { with inferior } \\
\text { vena cava thrombosis and/or } \\
\text { right atrium }\end{array}$ & $\begin{array}{l}\text { (radiotherapy OR "radiation therapy" OR EBRT) } \\
\text { AND ("hepatocellular carcinoma" OR HCC) AND } \\
\text { ("inferior vena cava" OR IVCT OR IVCTT) }\end{array}$ & 41 & None \\
\hline $\begin{array}{l}\text { Combined radiotherapy } \\
\text { (RT) with transarterial } \\
\text { chemoembolization (TACE) for } \\
\text { unresectable HCC }\end{array}$ & $\begin{array}{l}\text { (radiotherapy OR "radiation therapy" OR EBRT) } \\
\text { AND ("hepatocellular carcinoma" OR HCC) AND } \\
\text { ("transarterial chemoembolization" OR TACE) }\end{array}$ & 315 & 2004 \\
\hline $\begin{array}{l}\text { Combined RT with hepatic } \\
\text { arterial infusion chemotherapy } \\
\text { (HAIC) for unresectable HCC }\end{array}$ & $\begin{array}{l}\text { (radiotherapy OR "radiation therapy" OR EBRT) } \\
\text { AND ("hepatocellular carcinoma" OR HCC) AND } \\
\text { ("hepatic arterial infusion chemotherapy" OR HAIC) }\end{array}$ & 31 & 2017 \\
\hline $\begin{array}{l}\text { Stereotactic body radiotherapy } \\
\text { (SBRT) for HCC unsuitable for } \\
\text { conventional local treatment }\end{array}$ & $\begin{array}{l}\text { ("stereotactic body radiotherapy" OR SBRT) AND } \\
\text { ("hepatocellular carcinoma" OR HCC) }\end{array}$ & 164 & 2012 \\
\hline
\end{tabular}

\section{Publish your work in this journal}

OncoTargets and Therapy is an international, peer-reviewed, open access journal focusing on the pathological basis of all cancers, potential targets for therapy and treatment protocols employed to improve the management of cancer patients. The journal also focuses on the impact of management programs and new therapeutic agents and protocols on patient perspectives such as quality of life, adherence and satisfaction. The manuscript management system is completely online and includes a very quick and fair peer-review system, which is all easy to use. Visit http://www.dovepress.com/testimonials.php to read real quotes from published authors. 